\title{
Spontaneous Transection of a Retained 16 Year Old Nasogastric Tube
}

https://doi.org/10.47210/bjohns.2021.v29i1.394

\author{
Tanmoy Sarkar, ${ }^{1}$ Debabrata Biswas, ${ }^{1}$ Riya Das, ${ }^{1}$ Uday Shankar Roy ${ }^{1}$
}

\section{Introduction}

\section{ABSTRACT}

The insertion of nasogastric (NG) feeding tube or Ryle's tube is a common procedure for treating patients in different medical or surgical conditions. One of its indications is in patients who can't eat or swallow due to obstruction in upper digestive tract. Case Report

We encountered a 71 year old female patient with stricture in mid to low esophagus, who presented with a retained NG tube in situ for more than 16 years. Post admission, an NCCT scan of neck, thorax and upper abdomen showed about 30cm long retained tube with its lower end in the body of stomach. Upper gastro-intestinal endoscopy was subsequently performed and the retained tube was carefully removed in toto.

$\underline{\text { Discussion }}$

Insertion of nasogastric tube is a frequent and well tolerated day to day procedure though it can produce unexpected complications like stricture, perforation or haemorrhage and even spontaneous transection in a few patients with prolonged indwelling Ryle's tube. Long term placement of nasogastric tube is thus not recommended to avoid complications. $\underline{\text { Kevwords }}$ Tube, Nasogastric; Complications

$\mathrm{A}$ mongst various uses, nasogastric tube (NGT) is used for enteral feeding for the patients who present with stricture in upper digestive tract and provides access to the stomach for diagnostic and therapeutic purposes. Current recommendation, long term NGT should be changed every 4-6 weeks swapping to alternate nostril. ${ }^{1}$ There aren't many reported cases of retained / transected NGTs, probably any for such a long duration. The case illustrated is unique with respect to retention of NGT for 16 years, though the patient could swallow food or liquid per orally. Spontaneous transection of the tube, resulting in expulsion of the proximal part, with part of the NG tube in the esophagus and stomach being still retained, prompted the patient to seek medical attention.

\section{1 - R G Kar Medical College and Hospital. Kolkata}

\section{Corresponding author:}

Dr Debabrata Biswas

email: dr.dbiswas@hotmail.com

\section{Case Report}

A 71 year old female patient was admitted to Department of ENT through emergency department with retained NGT in esophagus. The NGT was present in situ allegedly, for last 16 years. The NGT had spontaneous transection on following which the patient rushed to the hospital. In 2004, i.e. 16 years back, the patient presented with dysphagia, subsequently the patient was diagnosed with esophageal ulceration and stricture at 30 $\mathrm{cm}$ from upper incisor, with inflamed ulcerated mucosa. Histopathological examination at that time suggested, "Epithelial hyperplasia with acute inflammatory changes" most probably due to persistent gastric reflux. Barium swallow also suggested "persistent narrowing of the lower part of esophagus with prestenotic dilatation".

The patient was managed conservatively along with 14 Fr NGT insertion for enteral feeding. (Fig 1) Thereafter, was lost follow up after one month and was allegedly doing well with the NGT in situ. The patient 


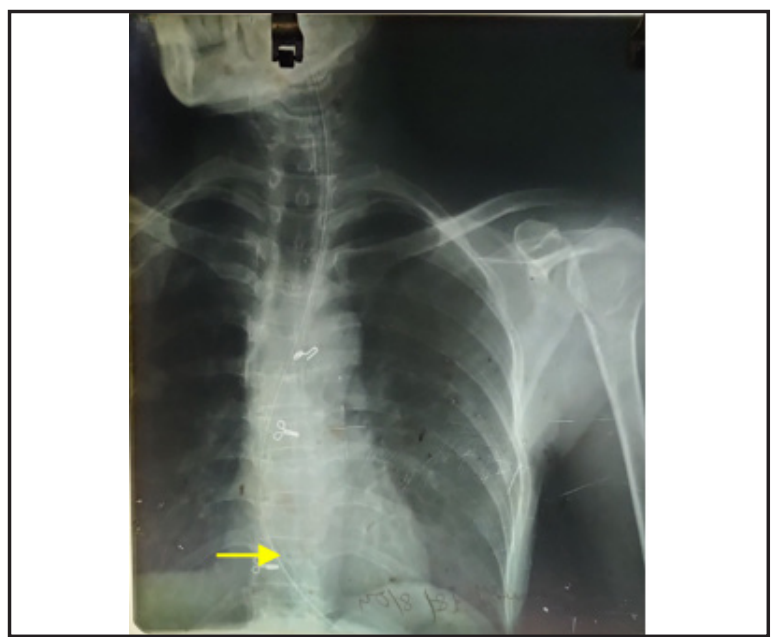

Fig. 1. NGT in situ in esophagus as shown in Chest X-ray PA View

began to take food and liquids perorally after one month and continued to do so though the NGT was there. After an uneventful period of 16 years, the patient had spontaneous transection of the NGT, following which the patient presented to us for further management. The mechanism of transection still remains unclear.

Post admission, basic blood investigations were all within normal limits. Chest X-ray (PA view) and X-ray abdomen (Erect posture) showed the presence of NGT from the hypopharynx upto the body of stomach. (Fig

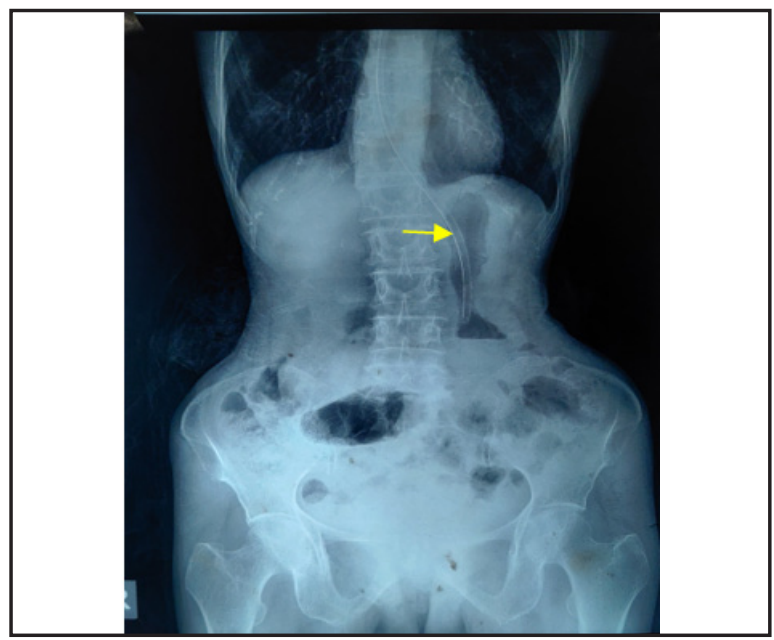

Fig. 2. Retained part of the NGT in stomach as shown in $x$-ray abdomen (yellow arrow head)s

2) A non-contrast computed tomography scan showed about $30 \mathrm{~cm}$ long retained tube with its lower end at the body of stomach. (Fig 3) The American Society for Gastrointestinal Endoscopy recommends urgent endoscopy for objects $>6 \mathrm{~cm}$ above proximal duodenum, which was performed in this case. ${ }^{2}$

Upper gastrointestinal endoscopy (UGIE) was subsequently performed on an emergency basis; the patient was awake while lubricating the flexible upper gastrointestinal endoscope with $2 \%$ lignocaine jelly. A

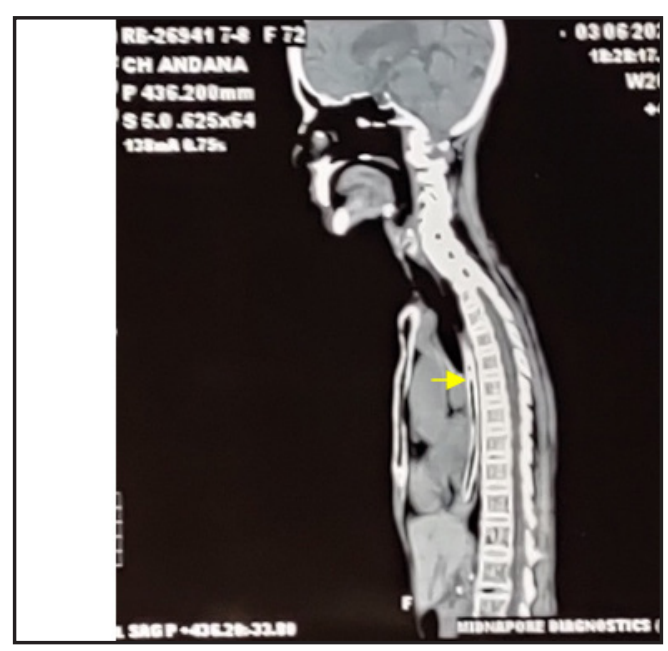

Fig. 3. Retained part of the NGT in esophagus as shown in NCCT thorax sagittal view (yellow arrow head)

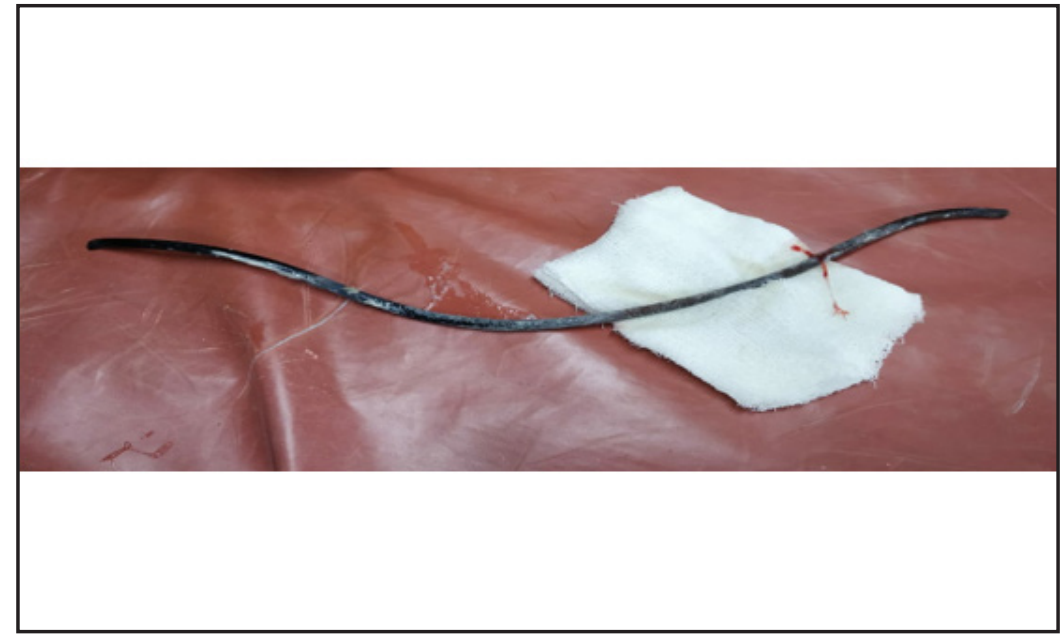

Fig. 4. Retained part of the NGT after removal. 
severed NGT was seen just distal to cricoids and it was carefully removed in toto using a rat toothed forceps (Fig 4). Gastro-esophageal junction was identified at 38 $\mathrm{cm}$ from upper incisor. Little areas of ulceration were seen at fundus, body, antrum and pylorus of the stomach most probably due to prolonged NG tube contact; 1st part of duodenum appeared healthy. Post-op period was uneventful. The patient was discharged on the following day.

\section{Discussion}

There are multiple indications for insertion of NGT in modern medicine, and commonly performed in the patients who need nutritional support as enteral feeding. It is generally a well tolerated process though it can produce unexpected complications occurring in $0.3-8 \%$ of patients. ${ }^{3}$ Long term tube placement can eventually lead to esophageal ulceration which may in turn result in stricture formation or perforation. Percutaneous gastrostomy is now preferred where long term NGT placement is anticipated.

There are very few studies to report the long term effects of NGT placement as nasogastric feeding is generally a short term intervention and only a few studies report use of it beyond 28 days. ${ }^{4}$ Another case reports spontaneous transection of NG tube after successful placement and 8 days of enteral feeding, ${ }^{5}$ but our case reports presence of the Ryle's tube in situ for 16 years following which the tube was spontaneously transected. Placement of NGT for longer durations is likely to increase the risks of irritation and erosion to the upper gastrointestinal tract as the tubes would eventually become rigid in due course time owing to regular exposure to low $\mathrm{pH}$ in the stomach. Stricture and hemorrhage may result from the inflammatory changes in upper gastrointestinal tract, and if severe may produce penetrating ulceration and esophageal perforation. ${ }^{6}$ Very long-term presence of NGT in situ can cause spontaneous transection of tube as was found in our case though it seems to be an extremely rare occurrence. So, it's prudent to adhere to the current recommendation to use the NGT in situ for a maximum period of seven days in case of small-bore tubes, and for wide bore tubes for a maximum period of two months.

\section{References}

1. Stroud M, Duncan H, Nightingale J. Guidelines for enteral feeding in adult hospital patients Gut 2003; 52:vii1-vii12

2. ASGE Standards of Practice Committee. Management of ingested foreign bodies and food impactions. Gastrointest Endosc. 2011; 73(6):1085-91

3. Jain Bhaskara Pillai, Annette Vegas, Stephanie Brister, Thoracic complications of nasogastric tube: review of safe practice. Interactive CardioVascular and Thoracic Surgery, Volume 4, Issue 5, October 2005, Pages 429-33

4. Dwolatzky T, Berezovski S, Friedmann R, et al. A prospective comparison of the use of nasogastric and percutaneous endoscopic gastrostomy tubes for long-term enteral feeding in older people. Clin Nutr. 2001;20:535-40

5. Cotter TG, Stier MW, Rao VL. Transected nasogastric tube: a rare complication of nasogastric feeding. Intern Emerg Med. 2019 Jan;14(1):179-180. doi: 10.1007/s11739-018-1928-5

6. Ahmed A, Aggarwal M, Watson E. Esophageal perforation: a complication of nasogastric tube placement. Am J Emerg Med. 1998;16:64-6. 